\title{
Apoio à decisão na seleção de investimentos em petróleo e gás: uma aplicação utilizando o método PROMETHEE
}

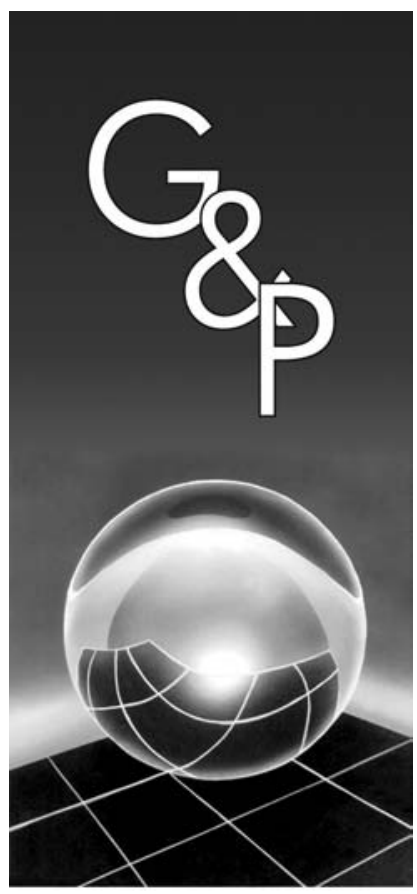

Afrânio Galdino de Araújo Adiel Teixeira de Almeida

\section{Resumo}

Este artigo apresenta uma aplicação da metodologia multicritério para seleção de investimentos estratégicos em Petróleo e Gás no nordeste do Brasil, usando o método PROMETHEE II. O método empregado no estudo foi selecionado a partir da modelagem das preferências dos decisores, da definição dos critérios utilizados e seus respectivos pesos. A aplicação numérica se apoia em informações do Planejamento Estratégico da Petrobrás para o período 2008-2012 e procura identificar o ordenamento completo das alternativas propostas, considerando as variáveis estratégicas e operacionais envolvidas no processo decisório.

Palavras-chave: Petróleo e gás. Método PROMETHEE. Decisão multicritério. Gestão estratégica. Investimentos.

\section{Introdução}

Decisão é o processo que leva - direta ou indiretamente à escolha de, ao menos, uma dentre várias diferentes alternativas, todas estas candidatas a resolver determinado problema (ALMEIDA, 2009; GOMES; GOMES; ALMEIDA, 2009). Além disso, o processo de tomada de decisão nos dias atuais tornou-se uma atividade de alta complexidade. Em função do aumento da velocidade das mudanças, dos elevados volumes de investimentos e do incremento do nível de incerteza nos ambientes competitivos, não é mais possível se determinarem as escolhas estratégicas de uma organização usando apenas ferramentas tradicionais. Portanto, a tomada de decisões estratégicas, em situações de incerteza nas organizações inseridas em ambientes caracterizados por rápidas mudanças, exige dos seus decisores melhores julgamentos, que possam melhor iluminar a escolha das alternativas em jogo (WRIGHT; KROLL; PARNELL, 2000).

A decisão acerca da alocação de recursos em um portfólio de projetos pode consumir muito tempo de trabalho, envolvendo normalmente a gerência de alto nível da organização, para a qual a escassez de tempo é fato notório (KEISLER, 2004). Especificamente, na seleção de projetos de investimentos em petróleo e gás, a escolha de alternativas estratégicas torna-se mais complexa em função da dependência de outros projetos de grande porte, que estão sendo desenvolvidos simultaneamente. O objetivo deste trabalho é fornecer aos decisores um apoio que ajude a esclarecer o problema em análise.

A cadeia produtiva do petróleo e gás, desde sua prospecção, passando pela exploração e a distribuição dos seus produtos e serviços, é considerada uma atividade de altos riscos e incertezas durante todo o seu processamento Além disso, a indústria do petróleo e gás tem registrado nos últimos anos um aumento significativo nos seus custos de exploração e produção.

As decisões a serem tomadas envolvem diversas variáveis econômicas, sociais, ambientais e políticas, além de recursos da ordem de bilhões de dólares; tais decisões demandam critérios variados na priorização dos projetos envolvidos. Há vários estudos na literatura recente mostrando o uso de métodos multicritério em problemas relacionados à cadeia produtiva do petróleo e gás (BRITO; DE ALMEIDA, 2009; MOTA; ALMEIDA; ALENCAR, 2009; BRITO; DE ALMEIDA, 2009).

Portanto, nessa indústria, a tomada de decisões é uma atividade complexa e que faz uso de métodos e técnicas para auxiliar os decisores a decidirem sob a influência de uma multiplicidade de critérios qualitativos e/ou quantitativos, 
muitas vezes conflitantes (GOMES; GOMES; ALMEIDA, 2009), buscando-se estabelecer uma relação subjetiva de preferências entre as alternativas que estão sendo avaliadas (ALMEIDA; COSTA, 2002). Esse é o dilema com que se defrontam os decisores quando da escolha de alocação de investimentos em petróleo e gás nas áreas estratégicas selecionadas pela Petrobrás.

De acordo com a estratégica corporativa desenvolvida pela Petrobrás para o seu Plano Estratégico 2008-2012, a empresa pretende ampliar sua atuação nos mercadosalvo de petróleo, derivados, petroquímico, gás e energia, biocombustíveis e distribuição, para se tornar referência mundial como uma empresa integrada de energia. Com isso, espera se tornar uma das cinco maiores empresas integradas de energia do mundo e a preferida pelo seu público de interesse (PETROBRÁS, 2007a).

Para alcançar as metas projetadas, o Plano de Negócio da Petrobrás para o período de 2008-2012 prevê investimentos de US\$ 112,4 bilhões, sendo 87\% (US\$ 97,4 bilhões) no Brasil e 13\% (US $\$ 15$ bilhões) no exterior, além de investir em projetos de exploração e refino em todo o Brasil. Estes investimentos garantem a consolidação da sustentabilidade da autossuficiência em óleo, LGN e gás natural nos próximos anos. As metas projetadas para 2012 estimam que a produção de petróleo e gás será de $3.058 \mathrm{mil}$ barris/dia de óleo equivalente por dia (boed) e de 3.455 mil barris/dia de óleo equivalente (boed) em 2015, (PETROBRÁS, 2007b).
A Bacia Potiguar faz do Rio Grande do Norte (RN) o segundo maior produtor de petróleo do Brasil e o primeiro na produção em terra com reservas estimadas em 600 MM boed (óleo equivalente por dia). Para o período de 2008-2012, a Petrobrás pretende realizar investimentos da ordem de US\$ 2 bilhões por ano para incrementar a extração, a produção e o refino de petróleo e gás natural. De 1976 até 2006 foram realizados investidos da ordem de 17 bilhões de dólares nas atividades terrestres e marítimas neste Estado.

No Rio Grande do Norte, a produção de óleo e LGN crescerá de 1.778 mil barris/dia em 2006 para 2.421 mil barris/dia em 2012. No tocante ao gás natural, a produção passará de $277 \mathrm{mil}$ barris/dia para $637 \mathrm{mil}$ barris/dia. A produção terá um crescimento médio de 7,6\% ao ano.

A infraestrutura da Petrobrás no RN conta com 5.590 poços em operação (um terço do total nacional), com 50 campos de exploração e 4.464 produtores, $556 \mathrm{~km}$ de oleodutos e $5.900 \mathrm{~km}$ de linhas de surgência, $542 \mathrm{~km}$ de gasodutos, 9 estações de tratamento e 32 plataformas marítimas. Isso garante uma exploração de petróleo da ordem de 106.600 barris/dia e de 36.000 barris/dia de gás, conforme mostra a Figura 1. Estes dados demonstram a importância da UN-RNCE na efetiva realização da estratégia corporativa definida pela Petrobrás.

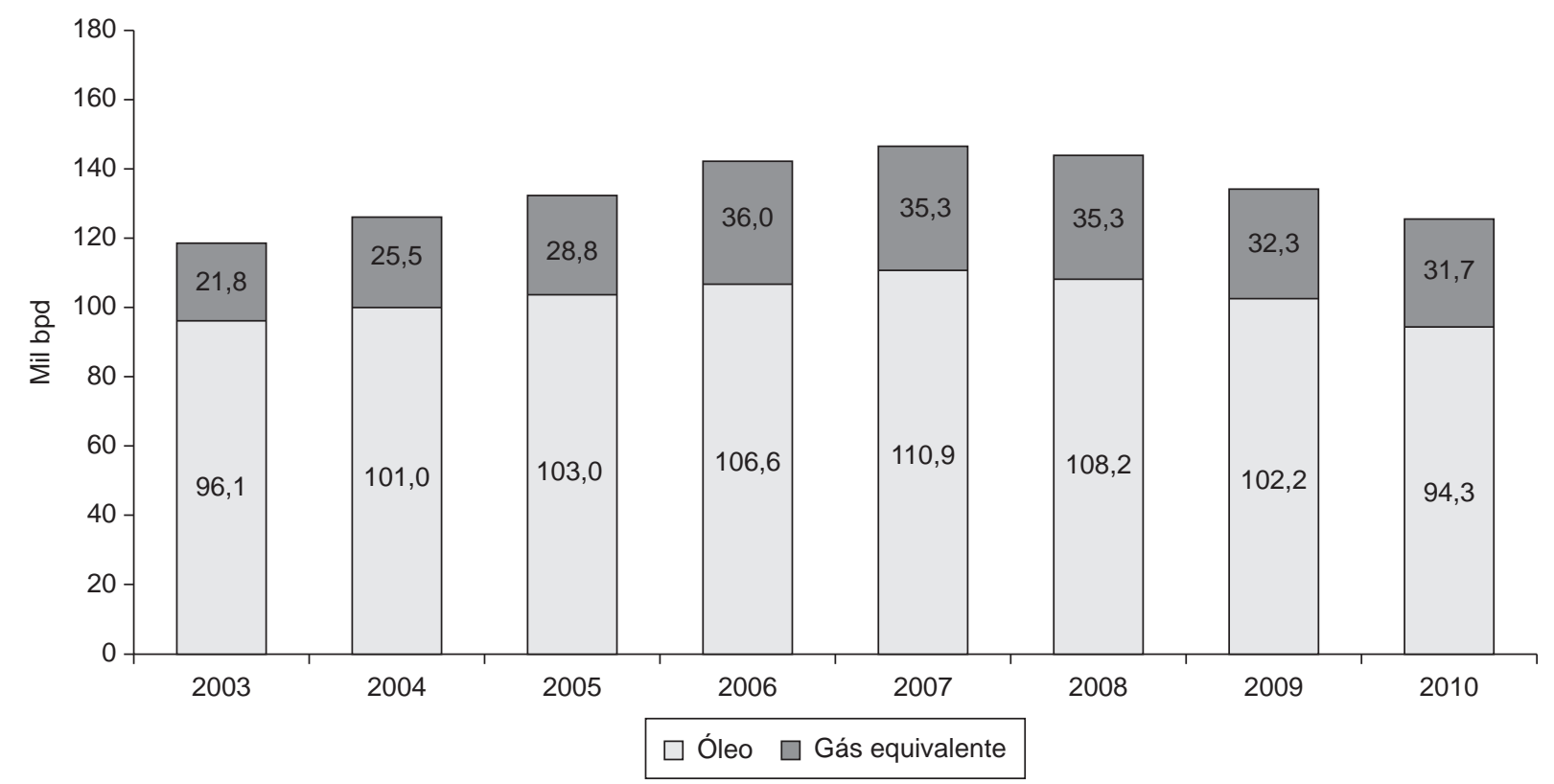

\begin{tabular}{|l|r|r|r|r|r|r|r|r|}
\hline & 2003 & 2004 & 2005 & 2006 & 2007 & 2008 & 2009 & 2010 \\
\hline Óleo & 96,1 & 101,0 & 103,1 & 106,5 & 110,9 & 108,2 & 102,2 & 94,3 \\
\hline Gás equivalente & 21,8 & 25,5 & 28,8 & 36,0 & 35,3 & 35,3 & 32,3 & 31,7 \\
\hline Total & 117,9 & 126,5 & 131,9 & 142,5 & 146,2 & 143,5 & 134,4 & 126,1 \\
\hline
\end{tabular}

Figura 1. Dados Setoriais RN (Petróleo e Gás). Fonte: Petrobrás/UN-RNCE, 2003. 


\section{Método PROMETHEE}

Uma das características mais relevantes dos seres humanos é a sua capacidade de tomar decisões. Às vezes, decisões simples e, em muitas vezes, decisões complexas. O processo de tomada de decisões dependerá de diversos fatores psicológicos, culturais e econômicos, além das habilidades e preferências do decisor e do conhecimento que estarão disponíveis no instante da tomada de decisões (ALMEIDA, 2009; GOMES; GOMES; ALMEIDA, 2009; FIGUEIRA; GRECO; EHRGOTT, 2005; ALMEIDA; COSTA, 2002; BELTON; STEWART, 2002; GOMES; ARAYA; CARIGNANO, 2004; ROY, 1996).

A problemática da tomada de decisão nos dias atuais é caracterizada por um número crescente de alternativas e critérios conflitantes, dentre os quais os decisores necessitam selecionar, ordenar, classificar ou ainda descrever com detalhes as alternativas a serem selecionadas, considerando múltiplos critérios.

Em função dessa complexidade, a metodologia Multicritério de Apoio à Decisão objetiva fornecer a quem necessita tomar uma decisão as ferramentas necessárias e suficientes para habilitá-lo nas soluções de problemas em que vários pontos de vista, até mesmo contraditórios, devem ser levados em consideração (ALMEIDA, 2009; VINCKE, 1992).

Portanto, a tomada de decisão pode ser definida como um esforço para resolver o dilema dos objetivos conflituosos, cuja presença impede a existência "solução ótima" e conduz à procura da "solução de melhor compromisso" (ZELENEY, 1982).

A problemática da decisão multicritério pode ser modelada com o apoio de várias metodologias que avaliam e selecionam alternativas à luz de múltiplos critérios, muitas vezes conflitantes.

Esta questão está também fortemente relacionada com os passos a serem seguidos no processo decisório, como: definição dos critérios, modelagem das preferências, estruturação do problema e a escolha do método de apoio à decisão a ser adotado (ALMEIDA, 2009; VINCKE, 1992).

A literatura é rica em métodos multicritério para apoio à tomada de decisão. Entre estes, os métodos da família PROMETHEE (Preference Ranking Method for Enrichment Evaluation), que objetivam construir relações de sobreclassificação de valores em problemas de tomada de decisão (VINCKE, 1992). Brans, Vincke e Mareschal (1986) apresentaram o método PROMETHEE como uma nova classe de métodos de sobreclassificação em análise multicritério. Suas principais características são simplicidade, clareza e estabilidade. A noção de critério generalizado é usada para construir uma relação de sobreclassificação valorada.

No processo de análise, se decompõe o objetivo em critérios e as comparações entre as alternativas são feitas no último nível de decomposição e aos pares, pelo estabelecimento de uma relação que acompanha as margens de preferência ditadas pelos agentes decisores (AL-RASHDAN et al., 1999).

A escolha de um dos métodos da família PROMETHEE para ser aplicado no presente estudo foi motivada pelo fato de estes métodos se destacarem dos demais por envolver conceitos e parâmetros com alguma interpretação física e econômica de fácil compreensão e assimilação pelos decisores. Além disso, esses métodos vêm passando por desenvolvimentos e adaptações complementares, que geraram as diversas metodologias da família PROMETHEE (BRANS; MARESCHAL, 2002). Outra questão que foi considerada está relacionada à abordagem não compensatória (ALMEIDA, 2009), que se mostrou a mais apropriada para o decisor envolvido neste problema.

Entretanto, de acordo com Brans e Mareschal (2002), uma das maiores dificuldades do uso do PROMETHEE pode ser a dificuldade do decisor em entender as funções de preferência, fato que funciona como uma barreira para seu melhor aproveitamento, uma vez que sua robustez é sobremaneira apoiada nos limites de indiferença e preferência. No caso deste estudo, isto não representou um problema, pois para o decisor o critério usual - que não requer os limites de indiferença e preferência - foi considerado apropriado.

De acordo com Vincke (1992), o decisor deve estabelecer para cada critério um peso $\left(\mathrm{p}_{\mathrm{j}}\right)$, que representa a sua importância relativa; o grau de sobreclassificação $\pi(a, b)$, para cada par de ações $(a, b)$, é calculado pelas Equações 1 e 2:

$$
\pi(a, b)=\frac{1}{P} \sum_{j=1}^{n} P_{j} F_{j}(a, b)
$$

em que:

$P=\sum_{j=1}^{n} P_{j}$ em que $F_{j}(a, b)$ é um número entre 0 e 1 .

$$
\begin{aligned}
& F_{j}(a, b)=0, \text { se } g_{j}(a) \leq g_{j}(b) e \\
& F_{j}(a, b)=1 \text { se } g_{j}(a)>g_{j}(b)
\end{aligned}
$$

Para estimar o valor de $\mathrm{F}_{\mathrm{j}}(\mathrm{a}, \mathrm{b})$, são oferecidas ao decisor seis formas de curvas para a escolha de qual é a melhor para a análise de cada critério, indicando a intensidade da preferência da diferença $g_{j}(a)-g_{j}(b)$. O Quadro 1 mostra as seis formas de critérios gerais para o PROMETHEE. Cada procedimento é feito de forma separada para cada critério.

O método PROMETHEE estabelece uma estrutura de preferência entre as alternativas discretas, tendo uma função de preferência entre as alternativas para cada critério. Essa função indica a intensidade da preferência de uma alternativa em relação à outra, com o valor variando entre 0 (indiferença) e 1 (preferência total).

Dentre as variações do PROMETHEE, o PROMETHEE I e o PROMETHEE II são as mais utilizadas e se 
diferenciam apenas pela forma de exploração da relação de sobreclassificação valorada.

\subsection{PROMETHEE I}

Na comparação entre as alternativas, consideram-se duas grandezas chamadas fluxo de importância positivo $\left(\Phi^{+}\right)$e fluxo de importância negativo $\left(\Phi^{-}\right)$. O primeiro fluxo (fluxo de saída) consiste em ordenar as ações seguindo uma ordem decrescente e representa o somatório dos índices de preferência de $a$ em relação a $b$ (Equação 3).

$$
\Phi^{+}(a)=\sum_{b \in a} \pi(a, b) / n-1
$$

O segundo fluxo $\left(\Phi^{-}\right)$, também chamado de fluxo de entrada, consiste em ordenar as ações de acordo com uma ordem crescente. Ele representa o somatório dos índices de preferência de $b$ em relação a $a$ (Equação 4).

$$
\begin{gathered}
\Phi^{-}(a)=\sum_{b \in a} \pi(b, a) / n-1 \\
\Phi(a)=\Phi^{+}(a)-\Phi^{-}(a)
\end{gathered}
$$

A Equação (5) fornece o chamado fluxo líquido de sobreclassificação e representa o balanço entre o poder e fraqueza da alternativa analisada. Quanto maior for $\Phi(a)$, melhor será a alternativa.
No método PROMETHEE I, a ordenação obtida provém da aceitação de uma possível relação de incomparabilidade entre as alternativas e tem como resultado final uma pré-ordem parcial. Conforme Olson (1996), a relação de sobreclassificação é definida da seguinte forma:

- $a \mathrm{P}^{+} b$ se e somente se $\Phi^{+}(a)>\Phi^{+}(b)$

- $a \mathrm{I}^{+} b$ se e somente se $\Phi^{+}(a)=\Phi^{+}(b)$

- $a \mathrm{P}^{-} b$ se e somente se $\Phi^{-}(a)<\Phi^{-}(b)$

- $a \mathrm{I}^{+} b$ se e somente se $\Phi^{-}(a)=\Phi^{-}(b)$

A alternativa $a$ sobreclassifica $b$, se:

$a \mathrm{P}^{+} b$ e $a \mathrm{P}^{-} b$ ou

$a \mathrm{P}^{+} b$ e $a \mathrm{I}^{-} b$ ou

$a \mathrm{I}^{+} b$ e $a \mathrm{P}^{-} b$

A alternativa $a$ é indiferente de $b$ se $a \mathrm{I}^{+} b$ e $a \mathrm{I}^{-} b$. As alternativas $a$ e $b$ são incomparáveis em todas as outras condições.

\subsection{PROMETHEE II}

Os cálculos do PROMETHEE II, de acordo com Olson (1996), resultam numa ordem completa das alternativas, terminando por perder algumas informações do PROMETHEE I, pois não admite a relação de incomparabilidade.

O método PROMETHEE II consiste em simplesmente ordenar as ações pelos números de $\Phi(a)$, desde que

\begin{tabular}{|c|c|c|}
\hline 1 - Critério usual não há parâmetro a ser definido & $\begin{array}{l}g_{\mathrm{f}}(a)-g_{\mathrm{f}}(b)>0 \\
g_{\mathrm{f}}(a)-g_{\mathrm{f}}(b) \leq 0\end{array}$ & $\begin{array}{l}F(a, b)=1 \\
F(a, b)=0\end{array}$ \\
\hline $\begin{array}{l}2 \text { - Quase-critério define-se o parâmetro q (limite de } \\
\text { indiferença) }\end{array}$ & $\begin{array}{l}g_{\mathrm{f}}(a)-g_{\mathrm{f}}(b)>q \\
g_{\mathrm{f}}(a)-g_{\mathrm{f}}(b) \leq q\end{array}$ & $\begin{array}{l}F(a, b)=1 \\
F(a, b)=0\end{array}$ \\
\hline \multirow[t]{2}{*}{$\begin{array}{l}3 \text { - Limite de preferência define-se o parâmetro p (limite } \\
\text { de preferência) }\end{array}$} & $\begin{array}{l}g_{\mathrm{f}}(a)-g_{\mathrm{f}}(b)>p \\
g_{\mathrm{f}}(a)-g_{\mathrm{f}}(b) \leq p \\
g_{\mathrm{f}}(a)-g_{\mathrm{f}}(b) \leq 0\end{array}$ & $g_{j}(a)-g_{j}(b)$ \\
\hline & & $F(a, b)=\frac{p}{F(a, b)=0}$ \\
\hline $\begin{array}{l}4 \text { - Pseudocritério definem-se os parâmetros q (limite de } \\
\text { indiferença) e p (limite de preferência) }\end{array}$ & $\begin{array}{l}\left|g_{\mathrm{f}}(a)-g_{\mathrm{f}}(b)\right|>p \\
q<\left|g_{\mathrm{f}}(a)-g_{\mathrm{f}}(b)\right| \leq p \\
\left|g_{\mathrm{f}}(a)-g_{\mathrm{f}}(b)\right| \leq q\end{array}$ & $\begin{array}{l}F(a, b)=1 \\
F(a, b)=1 / 2 \\
F(a, b)=0\end{array}$ \\
\hline $\begin{array}{l}5 \text { - Área de indiferença definem-se os parâmetros q } \\
\text { (limite de indiferença) e p (limite de preferência) }\end{array}$ & $\begin{array}{l}\left|g_{\mathrm{f}}(a)-g_{\mathrm{f}}(b)\right|>p \\
q<\left|g_{\mathrm{f}}(a)-g_{\mathrm{f}}(b)\right| \leq p \\
\left|g_{\mathrm{f}}(a)-g_{\mathrm{f}}(b)\right| \leq q\end{array}$ & $\begin{array}{l}F(a, b)=1 \\
F(a, b)=\left(\left|g_{\mathrm{f}}(a)-g_{\mathrm{f}}(b)\right|-q /(p-q)\right. \\
F(a, b)=0\end{array}$ \\
\hline $\begin{array}{l}6 \text { - Critério Gaussiano } \\
\text { O desvio-padrão deve ser fixado }\end{array}$ & $\begin{array}{l}g_{\mathrm{f}}(a)-g_{\mathrm{f}}(b)>0 \\
g_{\mathrm{f}}(a)-g_{\mathrm{f}}(b) \leq 0\end{array}$ & $\begin{array}{l}\text { A preferência aumenta segundo uma } \\
\text { distribuição normal } \\
F(a, b)=0\end{array}$ \\
\hline
\end{tabular}
(Equação 6):

Quadro 1. Critérios Gerais para o PROMETHEE. 


$$
\Phi(a)=\Phi^{+}(a)-\Phi^{-}(a)
$$

Escolhe-se, então, a alternativa que tenha o maior fluxo líquido de importância.

Dessa forma, o PROMETHEE II calcula os fluxos positivos e negativos da preferência para cada alternativa: o fluxo positivo significa quanto uma alternativa está dominando (poder) as outras e o fluxo negativo significa quanto uma alternativa é dominada (fraqueza) pelas outras.

Com base nesses fluxos, é obtido um ranking parcial por meio do PROMETHEE I. Por sua vez, o PROMETHEE II fornece um ranking completo, que se baseia no contrapeso dos dois fluxos de preferência. Os rankings desses dois métodos são influenciados pelos pesos alocados aos critérios. Existem outras variantes do PROMETHEE (III, IV e V), que tratam de situações mais complexas de decisão.

Este artigo apresenta uma aplicação numérica no processo de tomada de decisão estratégica em investimentos em petróleo e gás, na Bacia Sedimentar Potiguar, utilizando o software Decision Lab para o PROMETHEE II, que permite a obtenção de uma ordem completa das alternativas de um determinado cenário de negócios, quando se considera um conjunto de ações possíveis de serem realizadas.

\section{Aplicação numérica}

A seguinte aplicação numérica foi elaborada com base nas informações obtidas junto aos decisores da UN-RNCE, nos dados apresentados no Planejamento Estratégico 2008-2012 e no Plano de Negócio 2007-2011 da Petrobrás.

\subsection{Estruturação do problema}

O problema de decisão de investimentos em petróleo e gás apresenta características peculiares em relação a outros tipos de investimentos. Os principais vetores de decisão são os riscos políticos, operacionais, financeiros e tecnológicos, que fazem muitas vezes os decisores se defrontarem com situações em que têm de realizar comparações entre as possíveis consequências de suas decisões, necessitando algumas vezes abrir mão de excelentes condições operacionais para reduzir o risco político do investimento (MARGUERON; CARPIO, 2005).

Considerando a alta complexidade das decisões acerca de investimentos a serem realizados em Petróleo e Gás, a maior preocupação dos decisores é a alocação eficaz dos investimentos nas áreas escolhidas de forma compatível com os critérios que balizam as premissas de rentabilidade do plano de negócio com as exigências legais da área ambiental.

Uma das dificuldades encontrada na elaboração deste estudo foi a acessibilidade aos dados estratégicos da organização estudada. Não foi possível o pleno acesso a todos os agentes do processo de decisão durante a fase de entrevistas e levantamento de informações. Além disso, existe o sigilo das informações estratégicas para garantir o caráter de confidencialidade dos dados da organização; assim, foi necessário simular alguns dados para a efetiva realização dos objetivos propostos no estudo.

Para fins do estudo, o Rio Grande do Norte foi dividido em áreas para alocação dos investimentos e nomeadas de A1 a A7. Elas apresentam características peculiares no tocante à potencialidade de extração e exploração de petróleo e gás, e aos aspectos socioeconômicos da população, como IDH, qualidade de vida, investimentos governamentais, industriais, educacionais e serviços.

\subsection{Objetivos dos decisores}

- Selecionar e ordenar áreas que serão contempladas com novos investimentos no período de 2008-2012;

- Obter a melhor relação custo/benefício dos investimentos realizados em relação aos critérios estabelecidos pelos decisores estratégicos;

- Reduzir custos de extração (atualmente, da ordem de US\$ 3,60 por barril, média/bacia) no período estabelecido pelo planejamento estratégico, objetivando alcançar níveis internacionais de competitividade; e

- Reduzir perdas de exploração para valores estabelecidos nas métricas do planejamento estratégico para alcançar metas internacionais de competitividade.

\subsection{Contexto de atuação dos decisores}

- Determinação das áreas e dos níveis de investimentos que serão realizados durante o período de planejamento;

- Administração das pressões políticas originadas pelos agentes públicos e privados, que representam os stakeholders envolvidos na busca pela priorização dos investimentos nas regiões de seus interesses;

- Compatibilidade dos níveis de produtividade das áreas que podem afetar as decisões tanto em relação aos níveis de investimentos quanto ao timeline dos projetos em estudo; e

- Administração das pressões dos agentes ambientais para adequação dos projetos à legislação vigente.

\subsection{Escala de prioridade de investimentos (mínimo e máximo) por região}

- Em função da diversidade de processos técnicos, demanda mercadológica e logísticos utilizados nas várias áreas onde serão realizados os investimentos em exploração de petróleo e gás, serão adotados níveis diferentes de investimentos em cada uma delas. O Quadro 2 mostra o nível de prioridade dos investimentos por área estratégica. De acordo com as opiniões dos decisores, definiu-se uma escala de prioridade, considerando os níveis baixo, médio e alto de investimentos para alcançar as metas definidas no planejamento estratégico da Petrobrás para a UN-RNCE. 


\subsection{Restrições a serem consideradas pelos decisores}

\subsubsection{Definição dos critérios e seus respectivos pesos:}

De uma forma geral, os métodos de decisão multicritério apresentam uma demanda natural por critérios a partir dos quais a avaliação será realizada (BELTON; STEWART, 2002). Considera-se que os critérios estabelecidos devem satisfazer as propriedades de relevância de valor, compreensão, mensuralibilidade, não-redundância, independência de julgamento e balanceamento entre completude e concisão, dentre outras. De acordo com as percepções dos gestores entrevistados, o Quadro 3 mostra os critérios e seus respectivos pesos. Os pesos foram definidos a partir da experiência dos gestores entrevistados e apresentados em uma escala ordinal de 1 a 5 , na qual o valor 1 representa a menor importância dada ao critério e o valor 5 representa a maior importância atribuída ao critério.

\subsection{A Solução proposta}

Foram realizadas entrevistas com decisores, que emitiram seus juízos de valor em relação aos critérios e seus pesos e às preferências. A partir dos valores obtidos para as preferências das alternativas em relação aos critérios, o Quadro 4 apresenta a matriz em que são identificadas as intensidades de preferências dos decisores de cada alternativa em relação aos critérios escolhidos. Em face das respostas dos decisores - em que não houve dúvidas quanto à intensidade de suas preferências -, na utilização do método PROMETHEE II foi adotado o critério usual, segundo o qual não há parâmetros a serem definidos.

Os dados do Quadro 5 foram introduzidos no software Decision Lab 2000, versão 1.01. 0386, da empresa Visual Decision.

Quadro 2. Nível de Prioridade em Investimentos por Área Estratégica da UN-RNCE.

\begin{tabular}{|lccccccc|}
\hline $\begin{array}{l}\text { Áreas } \\
\text { estratégicas }\end{array}$ & A1 & A2 & A3 & A4 & A5 & A6 & A7 \\
$\begin{array}{l}\text { Nível de } \\
\text { investimentos }\end{array}$ & Alto & Médio & Alto & Baixo & Baixo & Baixo & Alto do Rodrigues \\
\hline
\end{tabular}

Quadro 3. Definição dos pesos dos critérios.

\begin{tabular}{|lcccccc|}
\hline Critérios & $\begin{array}{c}\text { Produtividade } \\
\text { da região }\end{array}$ & $\begin{array}{c}\text { Redução dos } \\
\text { custos de } \\
\text { exploração }(\%)\end{array}$ & $\begin{array}{c}\text { Redução das } \\
\text { perdas de } \\
\text { exploração }(\%)\end{array}$ & $\begin{array}{c}\text { Satisfação } \\
\text { dos agentes } \\
\text { governamentais }\end{array}$ & $\begin{array}{c}\text { Satisfação } \\
\text { dos agentes } \\
\text { ambientais }\end{array}$ & $\begin{array}{c}\text { Adequação às } \\
\text { especificações } \\
\text { técnicas }\end{array}$ \\
Pesos & 5 & 4 & 3 & 3 & 4 & 3 \\
Soma & & & 22 & & \\
\hline
\end{tabular}

Quadro 4. Matriz de avaliação de alternativas em relação aos critérios.

\begin{tabular}{|c|c|c|c|c|c|c|}
\hline \multirow[t]{3}{*}{ Áreas } & $\begin{array}{l}\text { Produtividade } \\
\text { da região }\end{array}$ & $\begin{array}{l}\text { Redução dos } \\
\text { custos de } \\
\text { exploração (\%) }\end{array}$ & $\begin{array}{c}\text { Redução das } \\
\text { perdas de } \\
\text { exploração (\%) }\end{array}$ & $\begin{array}{c}\text { Satisfação } \\
\text { dos agentes } \\
\text { governamentais }\end{array}$ & $\begin{array}{c}\text { Satisfação } \\
\text { dos agentes } \\
\text { ambientais }\end{array}$ & $\begin{array}{c}\text { Adequação às } \\
\text { especificações } \\
\text { técnicas }\end{array}$ \\
\hline & \multicolumn{6}{|c|}{ Pesos } \\
\hline & 5 & 4 & 3 & 3 & 4 & 3 \\
\hline A1 & 8 & 12 & 10 & 5 & 7 & 8 \\
\hline $\mathrm{A} 2$ & 7 & 15 & 7 & 6 & 6 & 7 \\
\hline A3 & 7 & 8 & 5 & 7 & 7 & 8 \\
\hline A4 & 5 & 5 & 15 & 3 & 4 & 6 \\
\hline A5 & 5 & 8 & 13 & 5 & 7 & 7 \\
\hline A6 & 6 & 7 & 8 & 4 & 6 & 6 \\
\hline A7 & 7 & 12 & 12 & 3 & 7 & 8 \\
\hline
\end{tabular}


Na Figura 2, é apresentado o ordenamento completo das alternativas estudadas e obtido por meio do comando View, opção Rankings do Decision Lab, com os fluxos líquidos $\Phi(a)=\Phi^{+}(a)-\Phi^{-}(a)$, que representam o balanço entre o poder e a fraqueza de cada alternativa analisada.

Utilizando o comando View, opção Flows, obtém-se o fluxo líquido de preferências multicritério para o estudo realizado, de acordo com o Quadro 6.

O comando View, opção Gaia Planes, do Decision Lab, fornece o correspondente Plano Gaia (Figura 3), que permite uma análise visual do problema de decisão estudado:

A alternativa A3 (Mar), que apresentou o maior fluxo líquido, se caracteriza por ser, dentre as alternativas estudadas, aquela que apresenta a maior distância em relação à origem, na direção do eixo de decisão $(\pi)$ e tendo seu desempenho melhor em relação aos critérios 3 e 4 . De forma análoga, pode-se observar que as alternativas A4 (Exploração), A5 (Mossoró) e A6 (Suporte), que obtiveram os piores lugares na classificação, estão situadas em pontos opostos à direção do referido eixo.

A existência de alternativas situadas em direções opostas no plano indica que existe a possibilidade de haver incomparabilidades, que não foram investigadas pelo

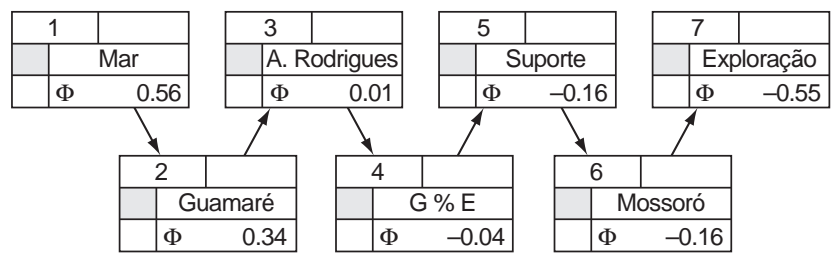

Figura 2. Ordenamento total das alternativas para o PROMETHEE II.

Quadro 6. Fluxos de preferências multicritério.

\begin{tabular}{|lccc|}
\hline & $\Phi+$ & $\Phi-$ & $\Phi$ \\
A. Rodrigues & 0.3712 & 0.3636 & 0.0076 \\
Exploração & 0.1818 & 0.7348 & -0.5530 \\
G\%E & 0.4167 & 0.4545 & -0.0379 \\
Guamaré & 0.5758 & 0.2348 & 0.3409 \\
Mar & 0.6591 & 0.0985 & 0.5606 \\
Mossoró & 0.3182 & 0.4773 & -0.1591 \\
Suporte & 0.3939 & 0.5530 & -0.1591 \\
\hline
\end{tabular}

Quadro 5. Dados do estudo no Decision Lab.

\begin{tabular}{|lcccccc|}
\hline & Critério 1 & Critério 2 & Critério 3 & Critério 4 & Critério 5 & Critério 6 \\
Min/Max & Maximizar & Minimizar & Minimizar & Maximizar & Maximizar & Maximizar \\
Peso & 5,00 & 4,00 & 3,00 & 3,00 & 4,00 & 3,00 \\
Função de preferência & Usual & Usual & Usual & Usual & Usual & Usual \\
Limiar de indiferença & - & - & - & - & - & - \\
Limiar de Preferência & - & - & - & - & - & - \\
Limiar Gaussiano & - & - & - & - & - & - \\
Unidade de Limiar & Absoluto & Absoluto & Absoluto & Absoluto & Absoluto & Absoluto \\
Performance média & 6,43 & 9,57 & 10,00 & 4,71 & 6,29 & 7,14 \\
Desvio padrão & 1,13 & 3,51 & 3,56 & 1,50 & 1,11 & 0,90 \\
Unidades & Produção Reg. & Red. C. E. \% & Red. P. E. \% & Sat. Ag. Gov. & Sat. Ag. Amb. & Ad. Esp. Tec. \\
Alto do Rodrigues & 7,00 & 12,00 & 12,00 & 3,00 & 7,00 & 8,00 \\
Exploração & 5,00 & 5,00 & 15,00 & 3,00 & 4,00 & 6,00 \\
G\%E & 7,00 & 15,00 & 7,00 & 6,00 & 6,00 & 7,00 \\
Guamaré & 8,00 & 12,00 & 10,00 & 5,00 & 7,00 & 8,00 \\
Mar & 7,00 & 8,00 & 5,00 & 7,00 & 7,00 & 8,00 \\
Mossoró & 5,00 & 8,00 & 13,00 & 5,00 & 7,00 & 7,00 \\
Suporte & 6,00 & 7,00 & 8,00 & 4,00 & 6,00 & 6,00 \\
\hline
\end{tabular}


PROMETHHE II e que são reforçadas pela posição do critério $\mathrm{C} 2$, quando comparado com relação à orientação dos critérios $\mathrm{C} 3$ e $\mathrm{C} 4$.

É importante ressaltar o valor de $\Delta=79,98 \%$, que é a medida da qualidade do gráfico do Plano Gaia. Como regra, se $\Delta$ for mais alto que $70 \%$, a qualidade do gráfico pode ser considerada como adequada. Para valores inferiores a 70\%, a interpretação do Plano Gaia deve ser feita com precaução.

A Figura 4, obtida por meio do comando View, opção Stability Intervals, no Decision Lab, apresenta os intervalos de estabilidade que dão para cada critério os limites dentro dos quais seus pesos podem ser modificados sem

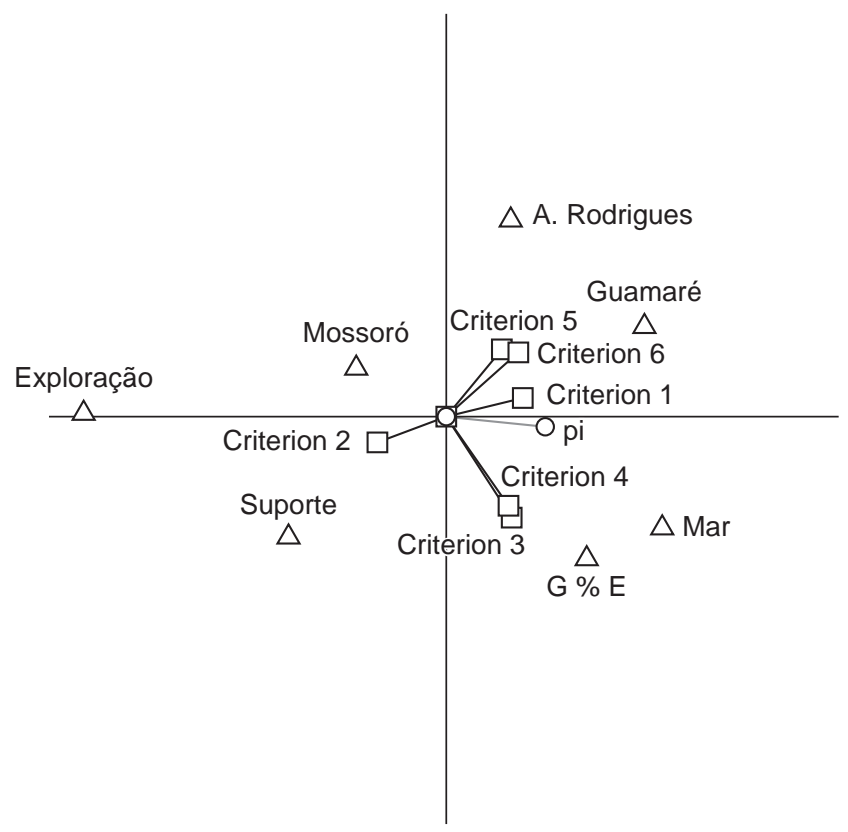

Figura 3. O plano gaia. mudar o ranking completo do PROMETHEE II. Foi feita uma análise de sensibilidade, na qual se variou os pesos dos critérios para $\pm 20 \%$ dos limites dos intervalos calculados.

Em todas as variações dos pesos testados, os fluxos líquidos $(\Phi)$ não foram significativamente alterados. Isso mostra uma tendência de pequenas alterações no ranking das alternativas de pouca importância. As variações dos valores dos fluxos não foram muito sensíveis às mudanças dos valores dos pesos.

Portanto, pode-se concluir que os resultados obtidos por meio dos pesos definidos na elaboração do estudo comportam-se de forma consistente quando são avaliados em relação às mudanças de cenários idealizados pelos decisores.

Finalmente, o Quadro 7 apresenta o ordenamento das alternativas estudadas de acordo com os resultados obtidos pelo Decision Lab para o PROMETHEE II. Os resultados encontrados apontam a alternativa A3 (Mar) como sendo a mais forte entre todas as outras.

Quadro 7. Ordenamento das alternativas.

\begin{tabular}{|ll|}
\hline \multicolumn{2}{|c|}{ Resposta PROMETHEE II: } \\
A3 - Mar & 0,5606 \\
A1 - Guamaré & 0,3409 \\
A7 - Alto do Rodrigues & 0,0076 \\
A2 - G \& E & $-0,0379$ \\
A6 - Suporte & $-0,1591$ \\
A5 - Mossoró & $-0,1591$ \\
A4 - Exploração & $-0,5530$ \\
\hline
\end{tabular}

Stability Level: 7 if first actions

\begin{tabular}{|c|c|c|c|c|c|c|}
\hline \multirow[b]{3}{*}{ Criterion1 } & \multirow[t]{2}{*}{ Weight } & \multicolumn{2}{|c|}{ Interval } & \multirow[t]{2}{*}{$\%$ Weight } & \multicolumn{2}{|c|}{$\%$ Interval } \\
\hline & & Min & $\operatorname{Max}$ & & Min & $\operatorname{Max}$ \\
\hline & 5.0000 & 5.0000 & 12.2500 & $22.73 \%$ & $22.73 \%$ & $41.88 \%$ \\
\hline Criterion2 & 4.0000 & 4.0000 & 5.6000 & $18.18 \%$ & $18.18 \%$ & $23.73 \%$ \\
\hline Criterion3 & 3.0000 & 3.0000 & 4.0000 & $13.64 \%$ & $13.64 \%$ & $17.39 \%$ \\
\hline Criterion4 & 3.0000 & 0.3333 & 3.0000 & $13.64 \%$ & $1.72 \%$ & $13.64 \%$ \\
\hline Criterions & 4.0000 & 3.0000 & 4.0000 & $18.18 \%$ & $14.29 \%$ & $18.18 \%$ \\
\hline Criterion6 & 3.0000 & 1.8000 & 3.0000 & $13.64 \%$ & $8.65 \%$ & $13.64 \%$ \\
\hline
\end{tabular}

Figura 4. Intervalos de estabilidade dos pesos dos critérios. 
Esse achado é compatível com os níveis históricos de investimentos nessa área, por se tratar de alternativa de grande importância estratégica em função da grande potencialidade de produção de petróleo e gás off-shore existente na região da Unidade RNCE.

As alternativas A3 (Mar), A1 (Guamaré) e A7 (Alto do Rodrigues) apresentam os maiores potenciais para investimentos em óleo e gás na Unidade RNCE e a ordem obtida no PROMETHEE I indicam essas áreas como prioritárias para investimentos na Bacia Potiguar. As demais alternativas ranqueadas, em função de suas importâncias relativas dentro do processo de planejamento estratégico, deverão ser contempladas com investimentos adequados às prioridades da Unidade RNCE.

\section{Conclusões}

O presente artigo apresenta uma aplicação numérica da metodologia PROMETHEE II, utilizando o software Decision Lab, em situações de incertezas e de altos riscos para a tomada de decisão em investimentos em petróleo e gás em áreas situadas na Bacia Potiguar.

O método PROMETHEE II foi escolhido pela adequação de suas características aos objetivos propostos pelos autores, permitindo a modelagem das preferências dos decisores de maneira bastante simples e de fácil entendimento. Além disso, o método atende de forma perfeita à problemática de escolha de alternativas, procurando esclarecer o problema para apoiar todo o processo de decisão.

Os resultados obtidos são compatíveis com a proposta dos autores de oferecer aos decisores um ranking completo de alternativas estratégicas que contribua para a melhoria do processo de tomada de decisão em portfólios de investimentos em Petróleo e Gás. Além disso, os autores recomendam que sejam realizados aperfeiçoamentos na metodologia empregada com a utilização de outros métodos multicritério, que permitem tratar de questões mais complexas, entre as quais as questões de maximização dos resultados obtidos para a otimização dos investimentos a serem realizados.

\section{Decision making in the selection of the investments in oil and gas: an application using the promethee method}

\section{Abstract}

This paper presents an application of multicriteria methodology in the selection of strategic investments in Oil and Gas in the northeast of Brazil, by using the PROMETHEE II method. The method used in this study was chosen as a result of modelling the decision-makers' preferences, the definition of the criteria to be used and their respective weights. The numerical application is supported by data from Petrobrás's Strategic Planning for the 2008-2012 period and attempts to identify the overall ranking of the proposed strategic alternatives, taking into consideration the strategic and operational variables involved in the decision process.

Key-words: oil and gas, PROMETHEE method, multicriteria decision, strategic management, investments.

\section{Referências bibliográficas}

ALMEIDA, A. T. Conhecimento e Uso de Métodos Multicritério de Apoio a Decisão. [S.1.]: Editora Universitária, 2009.

ALMEIDA, A. T.; COSTA, A. P. C. S. Modelo de decisão multicritério para priorização de sistemas de informação com base no método PROMETHEE. Gestão \& Produção, v. 9, n. 2, p. 201-214, Ago. 2002.

AL-RASHDAN, D. et al. Environmental impact assessment and ranking the environmental projects in Jordan. Theory and Methodology. European Journal of Operational Research, v. 118, p. 30-45, 1999.

BELTON, V.; STEWART, J. Multiple criteria decision analysis - an integrated approach. London: Kluver Academic Publishers, 2002 .
BRANS, J. P.; VINCKE, P.; MARESCHAL, B. How to select and how to rank projects: the PROMETHEE method. European Journal of Operational Research, v. 24, n. 2, p. 228-238, 1986.

BRANS, J. P.; MARESCHAL, B. PROMETHEE - GAIA: une méthodologie d'aide à La décision em présence de critères multiples. Bruxelles: Éditions de L'Université de Bruxelles, 2002.

BRITO, A. J; DE ALMEIDA, A. T. Multi-attribute risk assessment for risk ranking of natural gas pipelines. Reliability Engineering System and Safety, v. 94, p. 187-198, 2009.

BRITO, A. J.; DE ALMEIDA, A. T.; MOTA, C. M. M. A multicriteria model for risk sorting of natural gas pipelines based on electre tri integrating utility theory. European Journal of 
Operational Research, v. 200, p. 812-821, 2009. doi: 10.1016/j. ejor.2009.01.016

FIGUEIRA, J.; GRECO, S.; EHRGOTT, M. (Ed.). Multiple Criteria Decision Analysis: State of the Art Surveys. [S.1.]: Springer, 2005.

GOVERNO DO ESTADO DO RIO GRANDE DO NORTE. Secretaria de Estado do Meio Ambiente e dos Recursos Hídricos - SEMARH. Dados Setoriais 2004 -2007. Rio Grande do Norte, 2004.

GOMES, L. F. A. M.; GOMES, C. F. S.; ALMEIDA, A. T. de. Tomada de decisão gerencial: o enfoque multicritério. 3 ed. Rio de Janeiro: Ed. Atlas, 2009.

GOMES, L. F. A. M.; ARAYA, M. C. G.; CARIGNANO, C. Tomada de decisões em cenários complexos. Rio de Janeiro: Pioneira Thompson Learning, 2004.

MARGUERON, M. V.; CARPIO L. G. T. Processo de tomada de decisão sob incerteza em investimentos internacionais na exploração \& produção off-shore de petróleo: uma abordagem multicritério. Pesquisa Operacional, v. 25, n. 3, 2005.

MOTA, C. M. M.; ALMEIDA, A. T.; ALENCAR, L. H. A multiple criteria decision model for assigning priorities to activities in project management. International Journal of Project Management, v. 27, n. 2, p. 175-181, 2009.

OLSON, D. L. Decision aids for election problems. In: OLSON, D. L. Decision aids for election problems. New York: SpringerVerlag, 1996.

KEISLER, J. Value of information in portfolio. Decision analysis, v. 1, n. 4, p. 193-204, dez. 2004.

PETROBRÁS/UN RNCE - Dados Setoriais (Petróleo e Gás). Rio de Janeiro, 2003.

PETRÓLEO BRASILEIRO S/A - PETROBRÁS. Plano Estratégico 2008-2012. Rio de Janeiro, 2007a.

PETRÓLEO BRASILEIRO S/A - PETROBRÁS. Plano de Negócio 2008-2012. Rio de Janeiro, 2007b.

ROY, B. Multicriteria methodology for decision aiding. Netherlands: Kluver Academic Publishers, 1996.

WRIGHT, P. L.; KROLL, M. J.; PARNELL, J. Administração estratégica: conceitos. São Paulo: Atlas, 2000.

VINCKE, P. Multicriteria decision-aid. Bruxelles: John Wiley \& Sons, 1992.

ZELENY, M. Multiple criteria decision making. New York: MacGraw-Hill, 1982.

\section{Sobre os autores}

\section{Afrânio Galdino de Araújo}

Universidade Federal do Rio Grande do Norte

Av. Salgado Filho, 3000, CEP 59072-970, Natal - RN

e-mail: afranio.araujo@digi.com.br

\section{Adiel Teixeira de Almeida}

Programa de Pós-Graduação em Engenharia de Produção - PPGEP

Universidade Federal de Pernambuco

CP 7462, CEP 50630-970, Recife - PE

e-mail: almeidaatd@gmail.com

Agradecimentos: Este trabalho teve suporte parcial do CNPq e da CAPES. Os autores agradecem aos gestores estratégicos da UN-RNCE pelos dados e informações necessários à realização do estudo e também aos avaliadores anônimos, pelas suas contribuições em versão anterior a este trabalho, o que permitiu uma melhoria substancial da versão final. 